\title{
A STUDY ON THE DESIGN AND PERFORMANCE EVALUATION OF PNEUMATIC FIELDBUS SYSTEM
}

\author{
Dong Soo Kim*, Jeong Dai Jo* and Byung Oh Choi** \\ *Senior Researcher, IT Machinery Group, Advanced Industrial Technology Department \\ Korea Institute of Machinery and Materials \\ Yusung-Gu P.O Box 101, DAEJEON 305-343, KOREA \\ (E-mail* : kds671@kimm.re.kr), (E-mail* : micro@kimm.re.kr) \\ **Director, Advanced Industrial Technology Department \\ Korea Institute of Machinery and Materials \\ Yusung-Gu P.O Box 101, DAEJEON 305-343, KOREA \\ (E-mail : bochoi@kimm.re.kr)
}

\begin{abstract}
For pneumatic system controls, a data transmission system is needed with high speed and reliability for information interchange between main computer and 1/O devices. This paper presents a set of design techniques for a data communication system that is mainly used for pneumatic system controls. For this purpose, we first designed hardware modules for an interface between the central control module and the local node that handles the operation of solenoid valves. In addition, a communication protocol is developed for construction of RS-485 based multi-drop network, and this protocol is basically designed with a kind of polling technique. Finally we the performance of the developed system is evaluated. The field test results show that, even under high noise environment, the data transmission of $375 \mathrm{Kbps}$ rate is possible up to $1,000 \mathrm{~m}$ without using repeater. In addition, the system developed in this research can be easily extended for a communication network because of its modular structure.
\end{abstract}

\section{KEYWORDS}

Fieldbus, Data Transmission System, Pneumatic System, Communication Protocol, RS-485, Multi-Drop Network,

\section{INTRODUCTION}

For data interchange between controller and $\mathrm{I} / \mathrm{O}$ devices in pneumatic system, a data transmission system having high speed and reliability is required. Conventional method to construct the data transmission system is to use multi-cables between controller and $\mathrm{I} / \mathrm{O}$ devices. However, as the number of $\mathrm{I} / \mathrm{O}$ devices increase, the cost for implementation and maintenance increase dramatically. The fieldbus system solve such problems by using a single data bus instead of using multi-cables. In this system, all the data and command are transmitted through a single link and are used in time multiplexed form [1][2][3][4]. By using the fieldbus system, we can reduce the number of cables as well as modify number of field devices easily.

This research develops a data transmission system based on the filedbus structure, and applies the system for pneumatic system control. For this purpose, we first designed hardware modules for an interface between central control module and local node that handles the operation of solenoid valves. In addition we developed a communication protocol for construction of RS-485 based multi-drop 
network, and this protocol is basically designed with a kind of polling technique. Finally the performance of the developed system is evaluated. The field test results show that, even under high noise environment, the data transmission of $375 \mathrm{Kbps}$ rate is possible up to $1,000 \mathrm{~m}$ without using repeater. In addition, the system developed in this research is proved to be used easily for extension of a communication network because of its modular structure.

\section{SYSTEM DESIGN}

In the filedbus system, the local nodes are connected to a single data bus in parallel, and data communication are achieved by using the baseband method. In the baseband method, the modulation and demodulation devices are not used, and the maximum communication distance reaches to $1 \sim 2 \mathrm{Km}$. In addition, maximum 32 local nodes can be attached to a single segment. We can increase the maximum communication distance by using repeater. The signal transmitted from controller applies to valve/sensor terminal through the interface module, and make ON or OFF operations of the solenoid valve. In addition, the signal from limit switch mounted on solenoid valve are transmitted to controller, and the controller checks ON/OFF status of the solenoid valve.

The control system for fieldbus system consists of process controller and local node. The process controller consists of host control module and hardware interface circuit to PC. The local node consists of communication/power supply module, local node control module, and drive module.

\section{Host Control Module}

The host control module make interface for communication between the central controller (PC) and the local node, and the block diagram is described in Figure 1.

Solenoid Valves. As described in Figure 1, data communication between PC and local node isachieved by using the 8-bit microcomputer (Inte18751) and FIFO memory, and the FIFO memory works as a circular buffer. In addition, to minimize the noise generated from the communication lines, the transmitting/ receiving data using the microcomputer

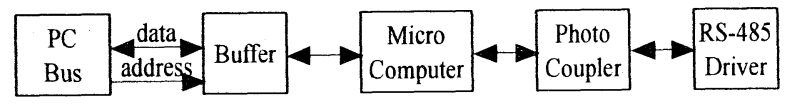

Figure 1. Block diagram of the host control module and the transmitting data through transmission lines are separated by using the photo-couplers. The data transmission rate (Baud rate) can be selected by setting the dip switches connected to microcomputer. In addition, the similar dip switches are used in the local node to select the data transmission rate. For normal data transmission, two dip switches must be set in the same position.

\section{Local Node}

As described previously, the local node consists of communication/power supply module, local node control module, and drive module, and the block diagram is described in Figure 2.

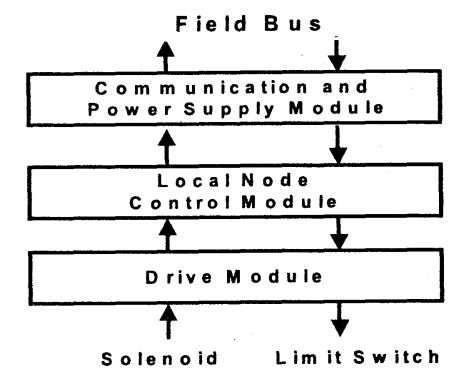

Figure 2. Block diagram of the local node.

\section{Communication/Power Supply Module}

The communication/power supply module work as two different devices. First, this module not only deliver data from the central controller to the local node control module but also deliver data from the local node control module to the central controller or another local nodes. Second, this module make two independent $+5 \mathrm{~V}$ power sources, $+5 \mathrm{~V}$ and $\mathrm{E} 5 \mathrm{~V}$, in which $\mathrm{E} 5 \mathrm{~V}$ is used as power for the photo-couplers connected to the data transmission line. By separating two power sources for communication and main control, we minimize the noise effects generated from the transmission lines.

\section{Local Node Control Module}

The local node control module work as an interface for communication between the central control module and the local node. In this research, we implement this module using 8-bits microcomputer, and it has very similar structure with the host control module. This module not only deliver data from the central control module to the drive module but also deliver data from the limit switch to the central control module.

In this module, we select data transmission rate by 


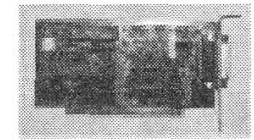

(a)

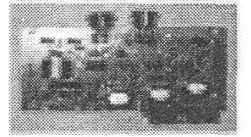

(10)

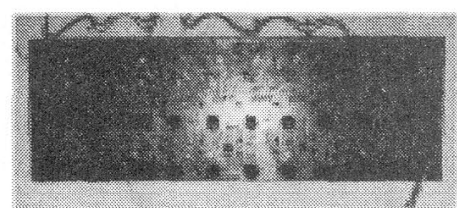

(d)

Figure 3 Picture of hardware module (a) Host control module (b) Communication/power supply module (c) Local node control module (d) Drive module

setting the dip switches connected to microcomputer, in which the data transmission rate must be the same as the rate that is set in the host control module. In addition, the address of the local node is selected by setting two switches connected to microcomputer.

\section{Drive Module}

The drive module work as two different devices. First, this module control the solenoid valve using data from the central control module. In this case, the transmitted data is serial form, and this data is converted to 8-bits parallel data. Using the parallel data, ON/OFF of 8 solenoids are controlled. Second, this module deliver data from the limit switches to the local node control module. In this case, data from the limit switches is 8-bits $p$ arallel data. After parallel data is converted to serial form, the data is transmitted to the local node control module. As described in Figure 3, since each module is implemented on independent board, the system is expanded easily.

\section{CONSTRUCTION OF RS -485 MULTI-DROP NETWORK}

The EIA RS-485 interface is a standard of transmission line which has bidirectional equivalence characteristics, and is widely used in industry. This standard is developed for multi-drop system, and has advantage of high data transmission rate in long distance environment. Each $\mathrm{I} / \mathrm{O}$ module operates independently, and use a single twisted pair as transmission media. Since each node can be connected to the network in parallel, new node can easily be added or removed without affecting system performance. When each node transmit or receive data, since they use simple command and response system, data collision is never occurred. In this case, there always exist a single central controller and multiple slave, and the central controller does not have address and the slave devices have different address. The command/response signal is transmitted from the central controller to designated slave device. In this case, the address of designated slave device is included in command. The designated slave device having the same address as the one from the controller execute the corresponding command, and the result can be checked in the central controller.

\section{Construction of RS -485 Network}

In this research, PC including host control module work as central controller and the local node control modules work as slave devices. When each local node control module does not transmit data, it becomes response state. We construct network using the daisychain method which can minimize signal reflection from transmission line[5]. In this method, the network is constructed with multiple segments which are connected with repeater. Total 32 local node control modules can be connected to each segment. When the number of module exceeds 32 , it leads to communication error due to rapid reduction of IC driver's current.

\section{Design of Communication Protocol}

The data communication between PC and the local nodes is executed by distinguishing transmitting data and receiving data. The transmitting data is used as control signals for solenoid valve or for receiving signals from limit switches. The receiving data is used for receiving data from limit switches. The transmitting data is consists of 5 fields ( 1 byte per field), and the receiving data is consists of 3 fields (1 byte per field). The pneumatic system is controlled by two different strategies. In the first case, to control the designated solenoid valve, set the value of ID and COMMAND to 0 , and put control signal into DATA 0 and DATA 1, and transmit this data through the bus. In this case, BCC data for error checking is automatically generated in the host control module by making logical EX-OR operation of value of ID, COMMAND, DATA 0 , and DATA 1. The local nodes connected to bus checks the value of ID, and reads the transmitted data when the 
value of ID is the same as its own address. In this case, since the value of COMMAND is set to 0 , DATA 0 and DATA 1 are used as control signal for solenoid valve. In addition, the local node prepare for error checking by taking logical operation. In other words, the local node computes the value of ERROR_CHECK $=$ (ID) EX-OR (COMMAND) EX-OR (DATA 0$)$ EX-OR (DATA 1). If ERROR_CHECK $=\mathrm{BCC}$, there are no errors in transmitted data. In contrast, if ERROR_CHECK $\neq$ $\mathrm{BCC}$, there are errors in transmitted data, and the controller should transmit the same data again.

In the second case, to check the status of limit switches attached to pneumatic system, set the value of ID and COMMAND to 1 , and transmit this data through the bus. In this case, since the data of DATA 0 and DATA 1 are not used as control signals, it does not affect the system operation. The local nodes connected to bus checks the value of ID, and reads the transmitted data when the value of ID is the same as its own address. In this case, since the value of COMMAND is set to 1 , the local node recognize that it has to transmit the status of limit switches to the central controller. The local node put the status of limit switches into DATA 0 and DATA 1 , and transmit this data to the controller. Then the central controller checks the status of limit switches using this data. In this case, BCC data for error checking is automatically generated in the local node module by naking logical EX-OR operation of value of DATA 0 and DATA 1 . In addition, the central controller prepare for error check by taking logical operation. In other words, the controller computes the value of ERROR_CHECK = (DATA 0) EX-OR (DATA 1). If ERROR_CHECK $=\mathrm{BCC}$, there are no errors in transmitted data from the local node. In contrast, if ERROR_CHECK $\neq \mathrm{BCC}$, there are errors in transmitted data, and the local node should transmit the same data again.

\section{PERFORMANCE EVALUATION}

We made performance evaluation with developed data transmission system, and Figure 6 shows facility for performance evaluation. Test items are data transmission rate, maximum distance, noise immunity, and reliability of control program. The test result shows that the developed system can be used for controlling solenoid valves, with data transmission rate of $375 \mathrm{Kbps}$ and up to distance of $1,000 \mathrm{~m}$ without using repeater. In addition, the developed system shows good noise characteristics by using two independent power sources for main control and communication. The probability of transmission error was also reduced by applying 1-byte checksum data.

\section{CONCLUSIONS}

In this research, we developed a data transmission system for pneumatic fieldbus system and designed hardware modules for interface between the central controller and pneumatic system. In addition, developed communication protocol for construction of RS-485 based multi-drop network. The performance result shows that the developed system can be used for controlling solenoid valves, with data transmission rate of $375 \mathrm{Kbps}$ and up to distance of $1,000 \mathrm{~m}$ without using repeater. In addition, the system can be used in highly noisy environment, and can easily be expanded. The system developed in this research can be applied to factory automation system and remote data acquisition system. For example, it could be possible to combine complex control system and make multiple distributed modules, and to construct a network in which all the modules are connected as subsystem. By applying this system, the data acquisition and information interchange between central controller and production lines become fast.

\section{REFERENCES}

1. M. Santori and K. Zech, "Fieldbus Brings Protocol to Process Control," IEEE Spectrum, pp. 60 - 64, March 1996

2. P. Pleinevaux and J. D. Decotignie, "Time Critical Communication Networks: Field Buses," IEEE Network, pp, 55 - 63, Vol. 2, No. 3, May 1988

3. J.G. Bollinger and N. A. Duffie, "Data-Driven Automation 4:Enabling Hardware," IEEE Spectrum, pp. 70 - 73, May 1983

4. K. Bender, PROFIBUS:The Fieldbus for Industrial Automation, Prentice Hall, NJ, 1993

5. IEEE Network Magazine:Special Issue on Communication for Manufacturing, Vol 2, No. 3, May 1988

6. W. Stallings, Data and Computer Communications, 4th edition, Prentice Hall, NJ, 1994

7. ISA-ds50.02, Standard:Fieldbus Standard for Use in Industrial Control Systems, Part 2:Physical Layer Specification and Services Definition, 1992. 